\title{
Corrigendum: Growth and modelling of spherical crystalline morphologies of molecular materials
}

O. Shalev, S. Biswas, Y. Yang, T. Eddir, O. Ahanotu, W. Lu, R. Clarke \& M. Shtein

Nature Communications 5:5204 doi: 10.1038/ncomms6204 (2015); Published 16 Oct 2014; Updated 3 Feb 2015

The authors wish to add Onye Ahanotu, who was originally included in the Acknowledgements, to the author list of this Article for contributing several samples and their electron micrographs. This has now been updated in both the PDF and HTML versions of the Article. 